\title{
A Survey on Distributed Filtering and Fault Detection for Sensor Networks
}

\author{
Hongli Dong, ${ }^{1,2}$ Zidong Wang, ${ }^{3,4}$ Steven X. Ding, ${ }^{2}$ and Huijun Gao ${ }^{5}$ \\ ${ }^{1}$ College of Electrical and Information Engineering, Northeast Petroleum University, Daqing 163318, China \\ ${ }^{2}$ Institute for Automatic Control and Complex Systems, University of Duisburg-Essen, 47057 Duisburg, Germany \\ ${ }^{3}$ School of Information Science and Technology, Donghua University, Shanghai 200051, China \\ ${ }^{4}$ Department of Information Systems and Computing, Brunel University, Uxbridge, Middlesex UB8 3PH, UK \\ ${ }^{5}$ Research Institute of Intelligent Control and Systems, Harbin Institute of Technology, Harbin 150001, China
}

Correspondence should be addressed to Zidong Wang; zidong.wang@brunel.ac.uk

Received 22 September 2013; Accepted 19 October 2013; Published 2 January 2014

Academic Editor: Xiao He

Copyright (C) 2014 Hongli Dong et al. This is an open access article distributed under the Creative Commons Attribution License, which permits unrestricted use, distribution, and reproduction in any medium, provided the original work is properly cited.

\begin{abstract}
In recent years, theoretical and practical research on large-scale networked systems has gained an increasing attention from multiple disciplines including engineering, computer science, and mathematics. Lying in the core part of the area are the distributed estimation and fault detection problems that have recently been attracting growing research interests. In particular, an urgent need has arisen to understand the effects of distributed information structures on filtering and fault detection in sensor networks. In this paper, a bibliographical review is provided on distributed filtering and fault detection problems over sensor networks. The algorithms employed to study the distributed filtering and detection problems are categorised and then discussed. In addition, some recent advances on distributed detection problems for faulty sensors and fault events are also summarized in great detail. Finally, we conclude the paper by outlining future research challenges for distributed filtering and fault detection for sensor networks.
\end{abstract}

\section{Introduction}

1.1. Sensor Networks. Sensor networks have recently been undergoing a quiet revolution in all aspects of the hardware implementation, software development, and theoretical research. In addition to the universal attributes of complex networks, sensor networks do possess their own characteristics due mainly to the large number of inexpensive wireless devices (nodes) densely distributed and loosely coupled over the region of interest. The past decade has seen successful applications of sensor networks in many practical areas ranging from military sensing, physical security, and air traffic control to distributed robotics and industrial and manufacturing automation. Accordingly, theoretical research on sensor networks has gained an increasing attention from multiple disciplines including engineering, computer science, and mathematics. Lying in the core part of the area are the distributed estimation and filtering problems that have recently been attracting growing research interests.

For distributed estimation/filtering problems, the inherently asynchronous sensor network is comprised of a large number of sensor nodes with computing and wireless communication capabilities, where the nodes are spatially distributed to form a wireless ad hoc network and every node has its own notion of time. Each individual sensor in a sensor network locally estimates/filters the system state from not only its own measurement but also its neighbouring sensors' measurements according to the given topology. The possible complexity of such a topology poses many challenges for scientists and engineers, and it is difficult to analyse these networks thoroughly with currently available estimation/filtering algorithms. Therefore, there is an urgent need to research on modelling, analysis of behaviours, systems theory, estimation, and filtering in sensor networks. Numerous fundamental questions have been addressed about the connections between sensor network topology and dynamic properties including stability, controllability, robustness, and other observable aspects. However, some major problems have not been fully investigated, such as the behaviour of stability, estimation, and filtering for sensor networks with incomplete/imperfect/stochastic topology, as well as their applications in, for example, distributed signal processing. 
Sensor networks have already become an ideal research area for control engineers, mathematicians, and computer scientists to manage, analyze, interpret, and synthesize functional information from real-world sensor networks. Sophisticated system theories and computing algorithms have been exploited or emerged in the general area of distributed sensor networks, such as analysis of algorithms, artificial intelligence, automata, computational complexity, computer security, concurrency and parallelism, data structures, knowledge discovery, DNA and quantum computing, randomisation, semantics, symbol manipulation, numerical analysis, and mathematical software. This survey aims to bring together the latest approaches to understanding, estimating, and filtering complex sensor networks in a distributed way. The references discussed in this paper include, but are not limited to the following aspects of sensor networks: (1) systems analysis of distributed sensor networks; (2) distributed parameter identification of sensor networks; (3) robustness and fragility analysis of distributed sensor networks; (4) methods and algorithms for sensor network dynamics; and (5) distributed estimation and filtering with limited communication constraints.

1.2. Distributed Filtering. Over the past ten years or so, the sensor networks (SNs) have proven to be a persistent focus of research attracting an ever-increasing attention in the areas of systems and communication. A typical sensor network is composed of a large number of spatially distributed autonomous sensor nodes and also a few control nodes, where each sensor has wireless communication capability as well as some level of intelligence for signal processing and for disseminating data [1-7]. The development of sensor networks was originally motivated by military applications such as distributed localization, power spectrum estimation, and target tracking problems. With recent intensive research in this area, sensor networks have a wide-scope domain of applications in areas such as environment and habitat monitoring, health care applications, traffic control, distributed robotics, and industrial and manufacturing automation $[1-3,8,9]$.

As one of the most fundamental collaborative information processing problems, the distributed filtering or estimation problem for sensor networks has gained particular concerns from many researchers and a wealth of the literature has appeared on this topic; see, for example, [10-19] and the references therein. For distributed filtering problems, the information available on an individual node of the sensor network is not only from its own measurement but also from its neighboring sensors' measurements according to the given topology. As such, the main difficulty in designing distributed filters lies in how to cope with the complicated coupling issues between one sensor and its neighboring sensors and how to reflect such couplings in the filter structure specification.

1.3. Distributed Fault Detection. On another research front, the fault detection problem has been an active field of research for the past decades because of the ever increasing demand for higher performance, higher safety, and reliability standards [20-33]. In sensor networks, sensor nodes have strong hardware and software restrictions in the light of processing power, memory capability, battery supply, and communication throughput, and faults are likely to occur frequently due to the low cost and the uncontrolled or even harsh environment where the sensor nodes are deployed. It is thus indispensable for the sensor networks to be able to detect, locate the faulty sensor nodes, and take actions to exclude them from the network during normal operation in order to ensure the network quality of service. Recently, some localized and distributed generic algorithms have been addressed in wireless sensor networks and a number of results about the distributed fault detection and fault tolerance have been published in the literature.

1.4. Structure of the Survey. The focus of this paper is to provide a timely review on the recent advances of the distributed filtering and fault detection issues for sensor networks. The rest of this paper is outlined as follows. In Section 2, the related results in the area of distributed filtering for wireless sensor networks are reviewed. The study contains a classification of different methods concerning distributed filtering. A comparison of different approaches is briefly summarized. Section 3 discusses the distributed fault detection problems over sensor networks. Both the distributed faulty sensors detection and distributed faultevent detection are carried out and explained separately. In Section 4, we give some concluding remarks and also point out some future directions.

\section{Distributed Filtering for Sensor Networks}

2.1. Traditional Kalman Filtering Approach. In recent years, the distributed filtering problem for sensor networks has received a fast growing research interest and some efficient distributed filtering/state estimation algorithms have been available in the literature; see, for example, [10, 11, 34-39] and the references therein.

The available algorithms, which can estimate stationary signals with low-cost and track nonstationary processes with reduced complexity, have a variety of engineering applications such as battlefield surveillance and target tracking. For example, a distributed Kalman filtering (DKF) algorithm has been introduced in [38] through which a crucial part of the solution is utilized to estimate the average of $n$ signals in a distributed way. Accordingly, this elegant algorithm has been developed in [34-36, 40,41] with different sensing models and dynamic consensus protocols. The notion of distributed bounded consensus filters has been introduced in [19] and the convergence analysis has been conducted for the corresponding distributed filters. In [14, 17, 42], the optimal distributed estimation algorithm has been proposed to adaptively update the weights for minimizing the estimated mean-square error. The diffusion-based Kalman filtering and smoothing algorithm has been established in $[10,11]$, where the information is diffused across the network through a sequence of Kalman iterations and data aggregation. In multisensor linear systems, several efficient algorithms including the centralised sensor fusion, distributed sensor fusion, and multialgorithm fusion to minimize the Euclidean estimation 
error of the state vector have been presented in $[43,44]$. In [45-47], the distributed particle filtering algorithm has been investigated as a response to offload the computation from the central unit as well as to reduce converge cast communication. References [48, 49] have introduced the maximum-likelihood approach in order to achieve the best possible variance for a given bandwidth constraint.

Looking into the issues discussed above, it can be observed that most available literature concerning the distributed filtering problems have been mainly limited to the traditional Kalman filtering theory that requires exact information about the plant model.

2.2. Robust and/or $H_{\infty}$ Filtering Approach. In the presence of modeling errors, parameter uncertainties, and external disturbance, it is difficult to ensure the robustness of the traditional Kalman filters especially when the unavoidable parameter drifts or external disturbances occur. Note that the robust performance of the available distributed filters has not been paid adequate research attention despite its clear engineering significance. In this sense, it is of great significance to include the robust and/or $H_{\infty}$ performance requirements for the distributed filtering problems.

Very recently, a new distributed $H_{\infty}$-consensus performance has been defined in [50] to quantify bounded consensus regarding the filtering errors over a finite horizon, the distributed filtering problem has been addressed for a class of linear time-varying systems in the sensor network, and the filter parameters have been designed recursively by resorting to the different linear matrix inequalities. The $H_{\infty}$-consensus performance presented in [50] has been utilized in [39] to deal with the distributed $H_{\infty}$ filtering problem for a class of polynomial nonlinear stochastic systems in sensor networks. Subsequently, the desired distributed $H_{\infty}$ filters have been designed in terms of the solution to certain parameterdependent linear matrix inequalities. A stochastic sampleddata approach has been addressed in [16] to investigate the distributed $H_{\infty}$ filtering in sensor networks. In [51], an $H_{\infty^{-}}$ type performance measure of disagreement between adjacent nodes of the network has been included and a robust filtering approach has been proposed to design the distributed filters for uncertain plants.

2.3. Filtering with Incomplete Information. It is worth noting that most reported results concerning the distributed filtering/estimation algorithms are for linear and/or deterministic systems. Since nonlinearities are ubiquitous in practice, it is necessary to consider the distributed filtering problem for target plants described by nonlinear systems. On the other hand, distributed filtering in a sensor network inevitably suffers from the constrained communication and computation capabilities that would degrade the network performances.

It is well known that, accompanied by the rapid development of network technologies, the network-induced phenomena have been thoroughly investigated for filtering and control problems of networked systems [13, 21, 5273]. Considering the case that the occurrence of incomplete information in sensor networks is more complex and severer due primarily to the network size, communication constraints, limited battery storage, strong coupling, and spatial deployment, the distributed filtering problem has been investigated in [74-76] for several classes of nonlinear stochastic systems over lossy sensor networks. The issue of average $H_{\infty}$ performance constraints has been brought up in [74], and then the distributed $H_{\infty}$ filtering problem has been investigated for system with repeated scalar nonlinearities and multiple probabilistic packet losses. Moreover, in [75], the distributed filtering problem has been further extended to the nonlinear time-varying systems with limited communication. The lossy sensor network suffers from quantization errors and successive packet dropouts that are described in a unified framework. A new distributed finite-horizon filtering technique by means of a set of recursive linear matrix inequalities has been proposed to satisfy the prescribed average filtering performance constraint.

In addition, the distributed $H_{\infty}$ filtering problem has been investigated in [76] for a class of discrete-time Markovian jump nonlinear time-delay systems with deficient statistics of modes transitions. In [77], a new approach has been proposed in virtue of the solvability of certain coupled recursive Riccati difference equations (RDEs) to deal with the distributed $H_{\infty}$ state estimation problem for a class of discrete time varying nonlinear systems with both stochastic parameters and stochastic nonlinearities.

\section{Distributed Fault Detection for Sensor Networks}

Wireless sensor networks (WSNs) are a multihop selforganized network system through wireless communication in which the failed nodes may decrease the service quality of the entire WSNs and create huge burden to the limited energy. In recent years, a growing number of efforts have been focused on the development of the fault detection methods for sensor nodes.

In [78], the online model-based detection of sensor faults has been first investigated by the cross-validation-based technique in which statistical methods are utilized to identify the sensors that are most likely to be faulty. This technique is centralized and can be applied to a broad set of fault models. A distributed fault detection scheme for sensor networks has been proposed in [79] to identify the faulty sensors, where each sensor node makes a decision based on the comparisons between its own sensing data and neighbors' data. The scheme, however, has the shortcoming of reducing the fault detection accuracy in the case that the number of neighbor's nodes to be diagnosed is small. In [80], an improved distributed fault detection algorithm based on weighted average value has been addressed by defining a new detection criterion to remedy the shortcoming that mentioned above. The scheme detects the sensor fault using spatial and time information simultaneously, where each sensor node identifies its own status based on local neighbor's average sensed data with some thresholds, hence maintaining low false alarm rate.

By using the spatial correlation of sensor measurements, a weighted median fault detection scheme has been introduced in [81] to detect the faults in WSNs. Reference [82] 
has studied the problem of designing a distributed faulttolerant decision fusion in the presence of sensor faults, where sensor fault detection scheme has been put forward to eliminate unreliable local decisions when performing distributed decision fusion. In [83], an agreement-based fault detection mechanism has been presented to detect clusterhead failures in clustered underwater sensor networks. Furthermore, a schedule generation scheme for a cluster head has been introduced to generate the transmission schedule of the forward and backward frames. The distributed fault detection problem has been investigated in [84] for WSNs, where each sensor node discerns its own status in view of local comparisons of sensed data with some thresholds and transfers the test results. It is well known that the basic idea of the distributed fault detection methods for sensor nodes is to check out the failed nodes by exchanging data and mutually testing among neighbor's nodes in this sensor networks.

It is worth pointing out that, apart from the development of distributed fault detection methods for sensor nodes, the distributed fault-event detection, which serves as a much more useful application in a sensor network, has also received much research attention. In [85], a distributed Bayesian fault recognition algorithm has been presented to solve the fault-event detection problem in sensor networks, where the randomized decision scheme and the threshold decision scheme have been used to derive analytical expressions for their detected performance. The proposed algorithm has the superiority of being completely distributed and localized each node by obtaining the information from neighboring sensors in order to make its decisions. A localized fault identification algorithm has been proposed in [86] to identify the faulty sensors and detect the reach of events in sensor networks, where each sensor node compares its own sensed data with the median of neighbors' data in order to determine its own status. In [87], a fault detection scheme for an event-driven wireless sensor network has been addressed by using an external manager, which can perform more complex functions compared to the sensor nodes. In [88], a fault-tolerant energy-efficient detection scheme has been presented to introduce the sensor fault probability into the optimal event detection process. For a given detection error bound, the minimum neighbors are selected to minimize the communication volume during the fault correction. It is also noted that the proposed distributed fault detection methods for sensor networks have a widely application fields such as the management of a reservoir [89] and integration of supply networks [90].

\section{Conclusions and Future Work}

In this paper, we have discussed and reviewed results, mostly from relatively recent work, on the problems of distributed filtering and fault detection for sensor networks. The various distributed filtering and fault detection technologies over sensor networks have been surveyed in great detail. Based on the literature review, some related topics for the future research work are listed as follows.

(i) A trend for future research is to generalize the methods obtained in the existing results to the distributed filtering and fault detection problems for nonlinear stochastic complex networks systems with randomly occurring incomplete information.

(ii) The nonlinearities considered in the existing results have some constraints that may bring somewhat conservative results. An additional trend for future research is to investigate the distributed filtering and fault detection problems for the general nonlinear systems for sensor networks.

(iii) Another future research direction is to further investigate the problems of nonparametric and robust sequential distributed detection for sensor networks.

(iv) The techniques such as conditional statistical tests and multivariate procedures in the presence of nonparametric hypotheses can be applied fruitfully in distributed fault detection applications.

\section{Conflict of Interests}

The authors declare that they have no conflict of interests related to this study.

\section{Acknowledgments}

This work was supported in part by the National Natural Science Foundation of China under Grants 61134009, 61329301, 61333012, 61374127 and 61004067, the Engineering and Physical Sciences Research Council (EPSRC) of the UK, the Royal Society of the UK, and the Alexander von Humboldt Foundation of Germany.

\section{References}

[1] A. Bertrand and M. Moonen, "Distributed adaptive nodespecific signal estimation in fully connected sensor networkspart I: sequential node updating," IEEE Transactions on Signal Processing, vol. 58, no. 10, pp. 5277-5291, 2010.

[2] A. Bertrand and M. Moonen, "Distributed adaptive nodespecific signal estimation in fully connected sensor networkspart II: simultaneous and asynchronous node updating," IEEE Transactions on Signal Processing, vol. 58, no. 10, pp. 5292-5306, 2010.

[3] P. Bianchi, J. Jakubowicz, and F. Roueff, "Linear precoders for the detection of a Gaussian process in wireless sensors networks," IEEE Transactions on Signal Processing, vol. 59, no. 3, pp. 882-894, 2011.

[4] E. Kokiopoulou and P. Frossard, "Distributed classification of multiple observation sets by consensus," IEEE Transactions on Signal Processing, vol. 59, no. 1, pp. 104-114, 2011.

[5] E. Kokiopoulou and P. Frossard, "Polynomial filtering for fast convergence in distributed consensus," IEEE Transactions on Signal Processing, vol. 57, no. 1, pp. 342-354, 2009.

[6] Y. Mostofi and M. Malmirchegini, "Binary consensus over fading channels," IEEE Transactions on Signal Processing, vol. 58, no. 12, pp. 6340-6354, 2010.

[7] A. Sundaresan and P. K. Varshney, "Location estimation of a random signal source based on correlated sensor observations," IEEE Transactions on Signal Processing, vol. 59, no. 2, pp. 787799, 2011. 
[8] B. Chen, K. Jamieson, H. Balakrishnan, and R. Morris, "Span: an energy-efficient coordination algorithm for topology maintenance in ad hoc wireless networks," in Proceedings of the 7th Annual International Conference on Mobile Computing and Networking, pp. 85-96, Rome, Italy, July 2001.

[9] R. Szewczyk, E. Osterweil, J. Polastre, M. Hamilton, A. Mainwaring, and D. Estrin, "Habitat monitoring with sensor networks," Communications of the ACM, vol. 47, no. 6, pp. 34-40, 2004.

[10] F. S. Cattivelli and A. H. Sayed, "Diffusion strategies for distributed Kalman filtering and smoothing," IEEE Transactions on Automatic Control, vol. 55, no. 9, pp. 2069-2084, 2010.

[11] F. S. Cattivelli and A. H. Sayed, "Diffusion strategies for distributed Kalman filtering: formulation and performance analysis," in Proceedings of the Cognitive Information Processing, pp. 36-41, Santorini, Greece, 2008.

[12] L. Chai, B. Hu, and P. Jiang, "Distributed state estimation based on quantized observations in a bandwidth constrained sensor network," in Proceedings of the 7th World Congress on Intelligent Control and Automation (WCICA '08), pp. 2411-2415, Chongqing, China, June 2008.

[13] H. Dong, Z. Wang, D. W. C. Ho, and H. Gao, "Varianceconstrained $H_{\infty}$ filtering for a class of nonlinear time-varying systems with multiple missing measurements: the finitehorizon case," IEEE Transactions on Signal Processing, vol. 58, no. 5, pp. 2534-2543, 2010.

[14] M. Farina, G. Ferrari-Trecate, and R. Scattolini, "Distributed moving horizon estimation for sensor networks," in Proceedings of the 1st IFAC Workshop on Estimation and Control of Networked Systems (NecSys '09), pp. 126-131, Venice, Italy, September 2009.

[15] J. Liang, Z. Wang, and X. Liu, "Distributed state estimation for discrete-time sensor networks with randomly varying nonlinearities and missing measurements," IEEE Transactions on Neural Networks, vol. 22, no. 3, pp. 486-496, 2011.

[16] B. Shen, Z. Wang, and X. Liu, "A stochastic sampled-data approach to distributed $H_{\infty}$ filtering in sensor networks," IEEE Transactions on Circuits and Systems I, vol. 58, no. 9, pp. 22372246, 2011.

[17] A. Speranzon, C. Fischione, K. Johansson, and A. SangiovanniVincentelli, "A distributed minimum variance estimator for sensor networks," IEEE Journal on Selected Areas in Communications, vol. 26, no. 4, pp. 609-621, 2008.

[18] J. Teng, H. Snoussi, and C. Richard, "Decentralized variational filtering for target tracking in binary sensor networks," IEEE Transactions on Mobile Computing, vol. 9, no. 10, pp. 1465-1477, 2010.

[19] W. Yu, G. Chen, Z. Wang, and W. Yang, "Distributed consensus filtering in sensor networks," IEEE Transactions on Systems, Man, and Cybernetics B, vol. 39, no. 6, pp. 1568-1577, 2009.

[20] S. X. Ding, T. Jeinsch, P. M. Frank, and E. L. Ding, "A unified approach to the optimization of fault detection systems," International Journal of Adaptive Control and Signal Processing, vol. 14, no. 7, pp. 725-745, 2000.

[21] H. Dong, Z. Wang, D. W. C. Ho, and H. Gao, "Robust $H_{\infty}$ filtering for Markovian jump systems with randomly occurring nonlinearities and sensor saturation: the finite-horizon case," IEEE Transactions on Signal Processing, vol. 59, no. 7, pp. 30483057, 2011.

[22] Z. Gao and S. X. Ding, "Actuator fault robust estimation and fault-tolerant control for a class of nonlinear descriptor systems," Automatica, vol. 43, no. 5, pp. 912-920, 2007.
[23] F. Gökas, Distributed control of systems over communication networks [Ph.D. dissertation], University of Pennsylvania, Philadelphia, Pa, USA, 2000.

[24] X. He, Z. Wang, and D. H. Zhou, "Robust fault detection for networked systems with communication delay and data missing," Automatica, vol. 45, no. 11, pp. 2634-2639, 2009.

[25] H. Dong, Z. Wang, and H. Gao, "Fault detection for Markovian jump systems with sensor saturations and randomly varying nonlinearities," IEEE Transactions on Circuits and Systems I, vol. 59, no. 10, pp. 2354-2362, 2012.

[26] H. Dong, Z. Wang, and H. Gao, "On design of quantized fault detection filters with randomly occurring nonlinearities and mixed time-delays," Signal Processing, vol. 92, no. 4, pp. 1117$1125,2012$.

[27] S. Yin, S. Ding, A. Haghani, H. Hao, and P. Zhang, "A comparison study of basic data-driven fault diagnosis and process monitoring methods on the benchmark Tennessee Eastman process," Journal of Process Control, vol. 22, no. 9, pp. 1567-1581, 2012.

[28] S. Yin, S. Ding, and H. Luo, "Real-time implementation of fault tolerant control system with performance optimization," IEEE Transactions on Industrial Electronics, vol. 61, no. 5, pp. 24022411, 2013.

[29] S. Yin, S. X. Ding, A. H. A. Sari, and H. Hao, "Data-driven monitoring for stochastic systems and its application on batch process," International Journal of Systems Science, vol. 44, no. 7, pp. 1366-1376, 2013.

[30] S. Ding, S. Yin, K. Peng, H. Hao, and B. Shen, "A novel scheme for key performance indicator prediction and diagnosis with application to an industrial hot strip mill," IEEE Transactions on Industrial Informatics, vol. 9, no. 4, pp. 2239-2247, 2012.

[31] S. Ding, P. Zhang, S. Yin, and E. Ding, "An integrated design framework of fault tolerant wireless networked control systems for industrial automatic control applications," IEEE Transactions on Industrial Informatics, vol. 9, no. 1, pp. 462-471, 2013.

[32] Y. Zheng, H. Fang, and H. O. Wang, "Takagi-Sugeno fuzzymodel-based fault detection for networked control systems with Markov delays," IEEE Transactions on Systems, Man, and Cybernetics B, vol. 36, no. 4, pp. 924-929, 2006.

[33] M. Zhong, S. X. Ding, and E. L. Ding, "Optimal fault detection for linear discrete time-varying systems," Automatica, vol. 46, no. 8, pp. 1395-1400, 2010.

[34] R. Carli, A. Chiuso, L. Schenato, and S. Zampieri, "Distributed Kalman filtering based on consensus strategies," IEEE Journal on Selected Areas in Communications, vol. 26, no. 4, pp. 622633, 2008.

[35] M. Kamgarpour and C. Tomlin, "Convergence properties of a decentralized Kalman filter," in Proceedings of the 47th IEEE Conference on Decision and Control (CDC '08), pp. 3205-3210, Cancun, Mexico, December 2008.

[36] R. Olfati-Saber, "Distributed Kalman filtering for sensor networks," in Proceedings of the 46th IEEE Conference on Decision and Control (CDC '07), pp. 5492-5498, New Orleans, La, USA, December 2007.

[37] R. Olfati-Saber, "Kalman-Consensus filter: optimality, stability, and performance," in Proceedings of the 48th IEEE Conference on Decision and Control held jointly with 28th Chinese Control Conference (CDC/CCC '09), pp. 7036-7042, Shanghai, China, December 2009.

[38] R. Olfati-Saber and J. S. Shamma, "Consensus filters for sensor networks and distributed sensor fusion," in Proceedings of 
the 44th IEEE Conference on Decision and Control, and the European Control Conference (CDC-ECC '05), pp. 6698-6703, Seville, Spain, December 2005.

[39] B. Shen, Z. Wang, Y. S. Hung, and G. Chesi, "Distributed $H_{\infty}$ filtering for polynomial nonlinear stochastic systems in sensor networks," IEEE Transactions on Industrial Electronics, vol. 58, no. 5, pp. 1971-1979, 2011.

[40] P. Alriksson and A. Rantzer, "Distributed Kalman filtering using weighted averaging," in Proceedings of the 17th International Symposium on Mathematical Theory of Networks and Systems, Kyoto, Japan, 2006.

[41] D. P. Spanos, R. Olfati-Saber, and R. M. Murray, "Approximate distributed kalman filtering in sensor networks with quantifiable performance," in Proceedings of the 4th International Symposium on Information Processing in Sensor Networks (IPSN '05), pp. 133-139, Piscataway, NJ, USA, April 2005.

[42] T.-Y. Wang, L.-Y. Chang, and P.-Y. Chen, "A Collaborative sensor-fault detection scheme for robust distributed estimation in sensor networks," IEEE Transactions on Communications, vol. 57, no. 10, pp. 3045-3058, 2009.

[43] T. M. Berg and H. F. Durrant-Whyte, "Model distribution in decentralized multi-sensor data fusion," in Proceedings of the American Control Conference, pp. 2292-2293, Boston, Mass, USA, June 1991.

[44] X. Shen, Y. Zhu, E. Song, and Y. Luo, "Minimizing Euclidian state estimation error for linear uncertain dynamic systems based on multisensor and multi-algorithm fusion," IEEE Transactions on Information Theory, vol. 57, no. 10, pp. 7131-7146, 2011.

[45] M. Coates, "Distributed particle filters for sensor networks," in Proceedings of the 3rd International Symposium on Information Processing in Sensor Networks (IPSN '04), pp. 99-107, New York, NY, USA, April 2004.

[46] M. Coates and G. Ing, "Sensor network particle filters: motes as particles," in Proceedings of the IEEE/SP 13th Workshop on Statistical Signal Processing, pp. 1152-1157, Novosibirsk, Russia, July 2005.

[47] H. Q. Liu, H. C. So, W. Chan, and K. W. K. Lui, "Distributed particle filter for target tracking in sensor networks," Progress in Electromagnetics Research, vol. 11, pp. 171-182, 2009.

[48] A. Ribeiro and G. B. Giannakis, "Bandwidth-constrained distributed estimation for wireless sensor networks-part I: gaussian case," IEEE Transactions on Signal Processing, vol. 54, no. 3, pp. 1131-1143, 2006.

[49] A. Ribeiro and G. B. Giannakis, "Bandwidth-constrained distributed estimation for wireless sensor networks-part II: unknown probability density function," IEEE Transactions on Signal Processing, vol. 54, no. 7, pp. 2784-2796, 2006.

[50] B. Shen, Z. Wang, and Y. S. Hung, "Distributed $H_{\infty}$-consensus filtering in sensor networks with multiple missing measurements: the finite-horizon case," Automatica, vol. 46, no. 10, pp. 1682-1688, 2010.

[51] V. Ugrinovskii, "Distributed robust filtering with $H_{\infty}$ consensus of estimates," Automatica, vol. 47, no. 1, pp. 1-13, 2011.

[52] H. Dong, Z. Wang, and H. Gao, "Robust $H_{\infty}$ filtering for a class of nonlinear networked systems with multiple stochastic communication delays and packet dropouts," IEEE Transactions on Signal Processing, vol. 58, no. 4, pp. 1957-1966, 2010.

[53] H. Gao, Z. Fei, J. Lam, and B. Du, "Further results on exponential estimates of Markovian jump systems with modedependent time-varying delays," IEEE Transactions on Automatic Control, vol. 56, no. 1, pp. 223-229, 2011.
[54] H. Gao and X. Li, " $H_{\infty}$ filtering for discrete-time state-delayed systems with finite frequency specifications," IEEE Transactions on Automatic Control, vol. 56, no. 12, pp. 2935-2941, 2011.

[55] H. Gao, T. Chen, and J. Lam, "A new delay system approach to network-based control," Automatica, vol. 44, no. 1, pp. 39-52, 2008.

[56] H. Gao and T. Chen, "A new approach to quantized feedback control systems," Automatica, vol. 44, no. 2, pp. 534-542, 2008.

[57] J. Hu, Z. Wang, H. Gao, and L. K. Stergioulas, "Extended Kalman filtering with stochastic nonlinearities and multiple missing measurements," Automatica, vol. 48, no. 9, pp. 20072015, 2012.

[58] J. Hu, Z. Wang, B. Shen, and H. Gao, "Quantised recursive filtering for a class of nonlinear systems with multiplicative noises and missing measurements," International Journal of Control, vol. 86, no. 4, pp. 650-663, 2013.

[59] J. Hu, Z. Wang, H. Gao, and L. K. Stergioulas, "Robust sliding mode control for discrete stochastic systems with mixed time delays, randomly occurring uncertainties, and randomly occurring nonlinearities," IEEE Transactions on Industrial Electronics, vol. 59, no. 7, pp. 3008-3015, 2012.

[60] X. Li and H. Gao, "Robust finite frequency $H_{\infty}$ filtering for uncertain 2-D Roesser systems," Automatica, vol. 48, no. 6, pp. 1163-1170, 2012.

[61] X. Li and H. Gao, "Robust finite frequency $H_{\infty}$ filtering for uncertain 2-D systems: the FM model case," Automatica, vol. 49, no. 8, pp. 2446-2452, 2013.

[62] B. Shen, Z. Wang, H. Shu, and G. Wei, "On nonlinear $H_{\infty}$ filtering for discrete-time stochastic systems with missing measurements," IEEE Transactions on Automatic Control, vol. 53, no. 9, pp. 2170-2180, 2008.

[63] B. Shen, Z. Wang, J. Liang, and Y. Liu, "Recent advances on filtering and control for nonlinear stochastic complex systems with incomplete information: a survey," Mathematical Problems in Engineering, vol. 2012, Article ID 530759, 16 pages, 2012.

[64] H. Dong, Z. Wang, X. Chen, and H. Gao, "A review on analysis and synthesis of nonlinear stochastic systems with randomly occurring incomplete information," Mathematical Problems in Engineering, vol. 2012, Article ID 416358, 15 pages, 2012.

[65] Z. Wang, D. W. C. Ho, H. Dong, and H. Gao, "Robust $H_{\infty}$ finite-horizon control for a class of stochastic nonlinear timevarying systems subject to sensor and actuator saturations," IEEE Transactions on Automatic Control, vol. 55, no. 7, pp. 1716$1722,2010$.

[66] Z. Wang, B. Shen, H. Shu, and G. Wei, "Quantized $H_{\infty}$ control for nonlinear stochastic time-delay systems with missing measurements," IEEE Transactions on Automatic Control, vol. 57, no. 6, pp. 1431-1444, 2012.

[67] Z. Wang, B. Shen, and X. Liu, " $H_{\infty}$ filtering with randomly occurring sensor saturations and missing measurements," Automatica, vol. 48, no. 3, pp. 556-562, 2012.

[68] Z. Wang, D. W. C. Ho, Y. Liu, and X. Liu, "Robust $H_{\infty}$ control for a class of nonlinear discrete time-delay stochastic systems with missing measurements," Automatica, vol. 45, no. 3, pp. 684-691, 2009.

[69] Z. Wang, Y. Liu, and X. Liu, " $H_{\infty}$ filtering for uncertain stochastic time-delay systems with sector-bounded nonlinearities," Automatica, vol. 44, no. 5, pp. 1268-1277, 2008.

[70] Z. Wang, Y. Wang, and Y. Liu, "Global synchronization for discrete-time stochastic complex networks with randomly occurred nonlinearities and mixed time delays," IEEE Transactions on Neural Networks, vol. 21, no. 1, pp. 11-25, 2010. 
[71] G. Wei, Z. Wang, and H. Shu, "Robust filtering with stochastic nonlinearities and multiple missing measurements," Automatica, vol. 45, no. 3, pp. 836-841, 2009.

[72] D. Yue, E. Tian, Y. Zhang, and C. Peng, "Delay-distributiondependent stability and stabilization of T-S fuzzy systems with probabilistic interval delay," IEEE Transactions on Systems, Man, and Cybernetics B, vol. 39, no. 2, pp. 503-516, 2009.

[73] H. Zhang, M. Li, J. Yang, and D. Yang, "Fuzzy model-based robust networked control for a class of nonlinear systems," IEEE Transactions on Systems, Man, and Cybernetics A, vol. 39, no. 2, pp. 437-447, 2009.

[74] H. Dong, J. Lam, and H. Gao, "Distributed $H_{\infty}$ filtering for repeated scalar nonlinear systems with random packet losses in sensor networks," International Journal of Systems Science, vol. 42, no. 9, pp. 1507-1519, 2011.

[75] H. Dong, Z. Wang, and H. Gao, "Distributed filtering for a class of time-varying systems over sensor networks with quantization errors and successive packet dropouts," IEEE Transactions on Signal Processing, vol. 60, no. 6, pp. 3164-3173, 2012.

[76] H. Dong, Z. Wang, and H. Gao, "Distributed $H_{\infty}$ filtering for a class of Markovian jump nonlinear time-delay systems over lossy sensor networks," IEEE Transactions on Industrial Electronics, vol. 60, no. 10, pp. 4665-4672, 2013.

[77] D. Ding, Z. Wang, H. Dong, and H. Shu, "Distributed $H_{\infty}$ state estimation with stochastic parameters and nonlinearities through sensor networks: the finite-horizon case," Automatica, vol. 48, no. 8, pp. 1575-1585, 2012.

[78] F. Koushanfar, M. Potkonjak, and A. Sangiovanni-Vincentelli, "On-line fault detection of sensor measurements," in Proceedings of the 2nd IEEE International Conference on Sensors, vol. 2, pp. 974-979, October 2003.

[79] J. Chen, S. Kher, and A. Somani, "Distributed fault detection of wireless sensor networks," in Proceedings of the Workshop on Dependability Issues in Wireless Ad Hoc Networks and Sensor Networks (DIWANS '06), pp. 65-71, New York, NY, USA, September 2006.

[80] S. Ji, S.-F. Yuan, T.-H. Ma, and C. Tan, "Distributed fault detection for wireless sensor based on weighted average," in Proceedings of the 2nd International Conference on Networks Security, Wireless Communications and Trusted Computing (NSWCTC '10), vol. 1, pp. 57-60, Wuhan, China, April 2010.

[81] J.-L. Gao, Y.-J. Xu, and X.-W. Li, "Weighted-median based distributed fault detection for wireless sensor networks," Journal of Software, vol. 18, no. 5, pp. 1208-1217, 2007.

[82] T.-Y. Wang, L.-Y. Chang, D.-R. Duh, and J.-Y. Wu, "Distributed fault-tolerant detection via sensor fault detection in sensor networks," in Proceedings of the 10th International Conference on Information Fusion, pp. 1-6, Quebec, Canada, July 2007.

[83] P. Wang, J. Zheng, and C. Li, "An agreement-based fault detection mechanism for underWater sensor networks," in Proceedings of the 50th Annual IEEE Global Telecommunications Conference (GLOBECOM '07), pp. 1195-1200, Washington, DC, USA, November 2007.

[84] M.-H. Lee and Y.-H. Choi, "Fault detection of wireless sensor networks," Computer Communications, vol. 31, no. 14, pp. 3469$3475,2008$.

[85] B. Krishnamachari and S. Iyengar, "Distributed Bayesian algorithms for fault-tolerant event region detection in wireless sensor networks," IEEE Transactions on Computers, vol. 53, no. 3, pp. 241-250, 2004.

[86] M. Ding, D. Chen, K. Xing, and X. Cheng, "Localized faulttolerant event boundary detection in sensor networks," in
Proceedings of the 24th Annual Joint Conference of the IEEE Computer and Communications Societies, vol. 2, pp. 902-913, March 2005.

[87] L. B. Ruiz, I. G. Siqueira, L. B. Oliveira, H. C. Wong, J. M. S. Nogueira, and A. A. F. Loureiro, "Fault management in eventdriven wireless sensor networks," in Proceedings of the 7th ACM Symposium on Modeling, Analysis and Simulation of Wireless and Mobile Systems, pp. 149-156, New York, NY, USA, October 2004

[88] X. Luo, M. Dong, and Y. Huang, "On distributed fault-tolerant detection in wireless sensor networks," IEEE Transactions on Computers, vol. 55, no. 1, pp. 58-70, 2006.

[89] Y. Chen and K. A. Hoo, "Stability analysis for closed-loop management of a reservoir based on identification of reduced-order nonlinear model," Systems Science and Control Engineering, vol. 1, no. 1, pp. 12-19, 2013.

[90] S. R. Desai and R. Prasad, "A new approach to order reduction using stability equation and big bang big crunch optimization," Systems Science and Control Engineering, vol. 1, no. 1, pp. 20-27, 2013. 


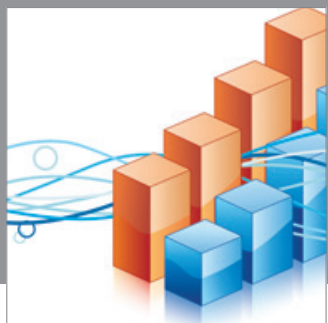

Advances in

Operations Research

mansans

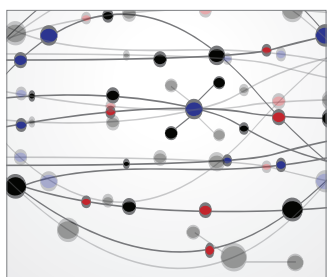

The Scientific World Journal
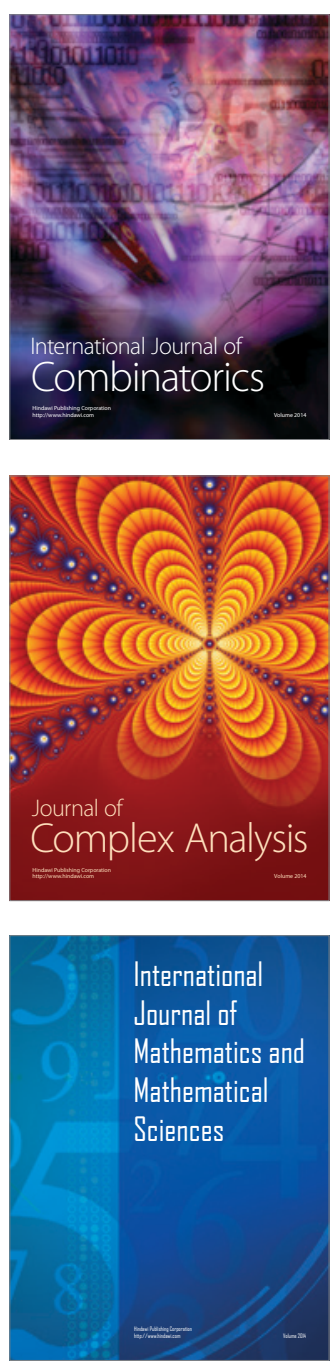
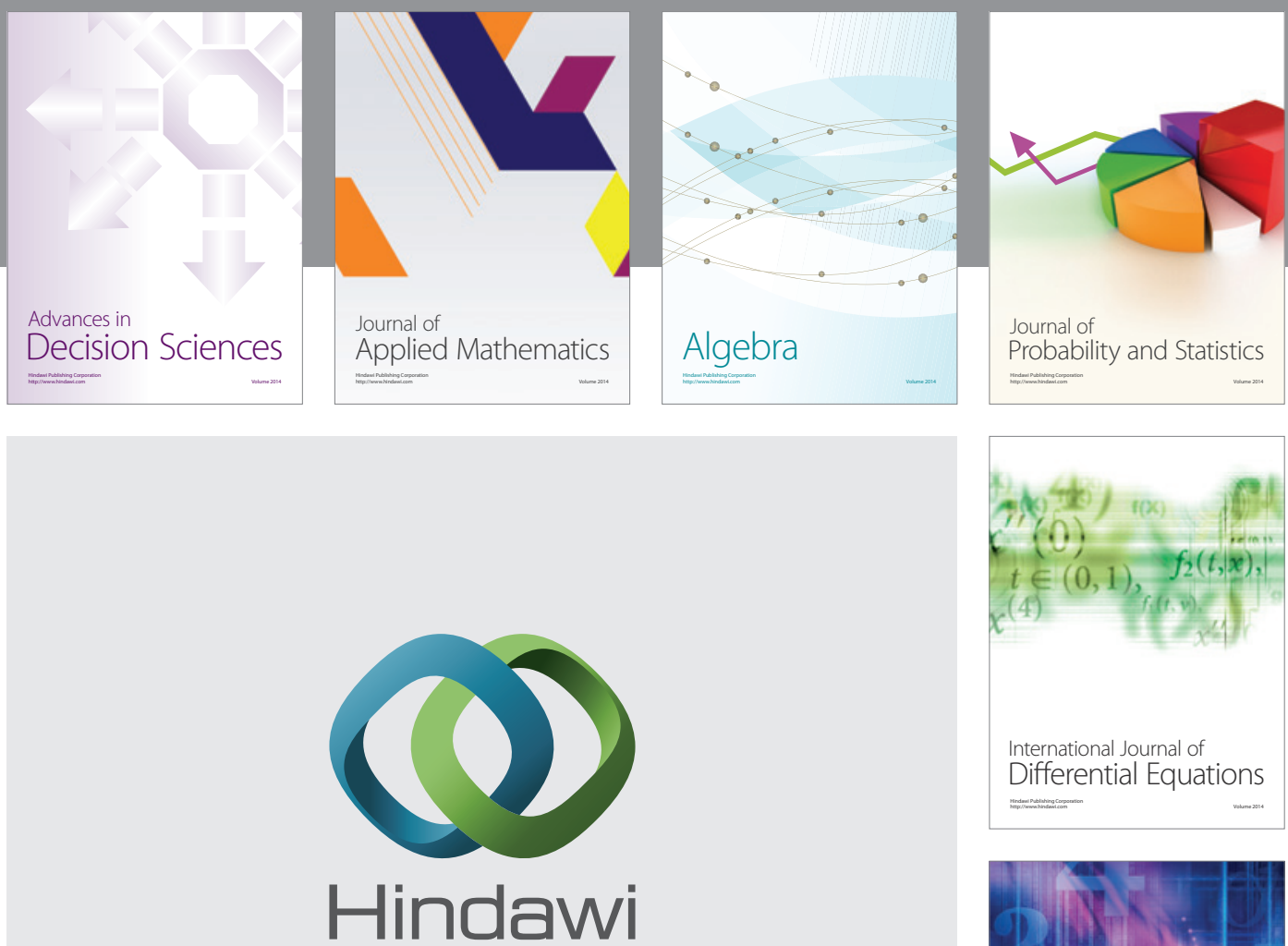

Submit your manuscripts at http://www.hindawi.com
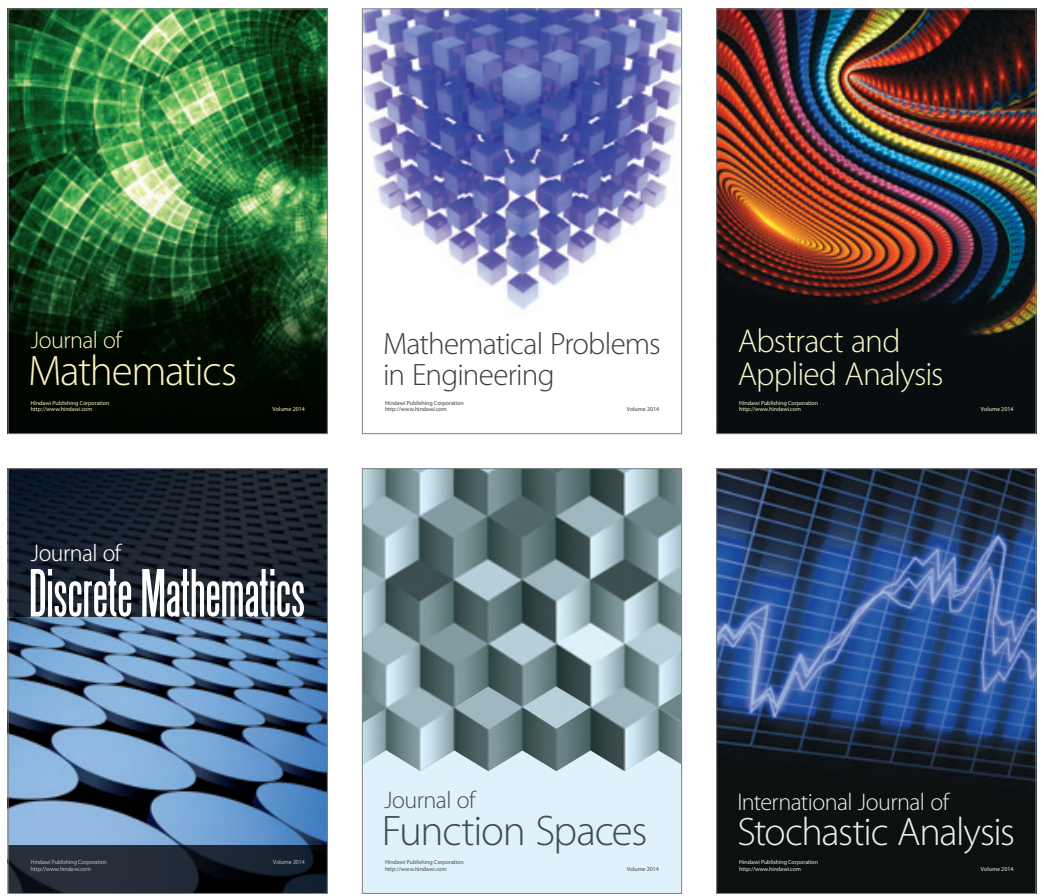

Journal of

Function Spaces

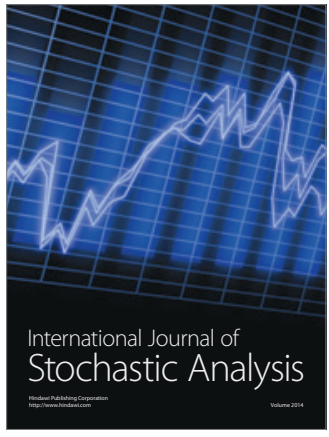

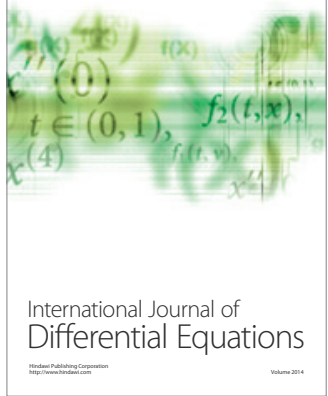
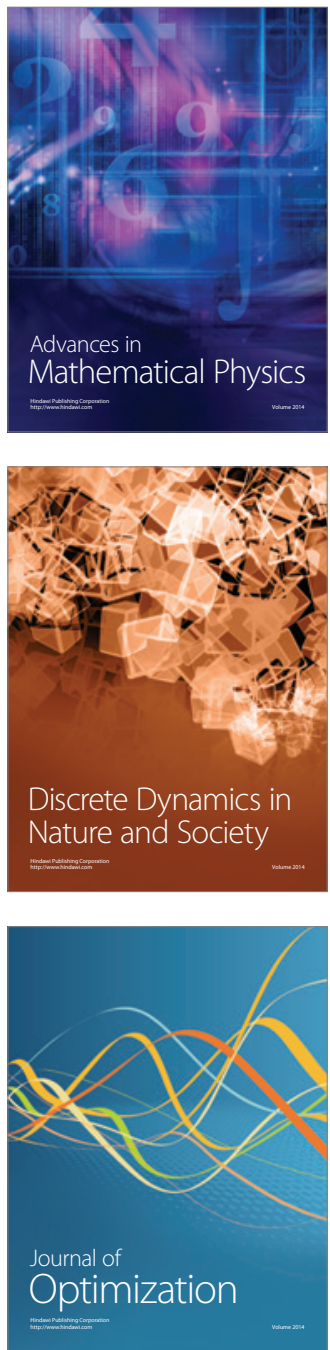